\title{
Prevalence of Candida albicans and non- albicans on the tongue dorsa of elderly people living in a post-disaster area: a cross-sectional survey
}

Toshiro Sato ${ }^{1}$, Mitsuo Kishi ${ }^{1 *}$, Miki Suda ${ }^{1}$, Kiyomi Sakata$^{2}$, Haruki Shimoda ${ }^{2}$, Hiroyuki Miura ${ }^{3}$, Akira Ogawa ${ }^{4}$ and Seiichiro Kobayashi ${ }^{4}$

\begin{abstract}
Background: Candida species are normal commensal organisms of the mouth. However, they can cause oral mucosal and severe systemic infections in persons with reduced immune function, which is common in the very elderly. In post-disaster areas, the number of elderly residents rapidly increases due to the outflow of younger generations. Hence, we examined the prevalence of Candida albicans and non-albicans in association with oral and systemic conditions, life style, medications, and living conditions.

Methods: This study was performed in 2014. Participants of this study were 266 community dwellers aged 60 years or older in Otsuchi town, which was severely damaged by the Great East Japan Earthquake and Tsunami in 2011. Oral

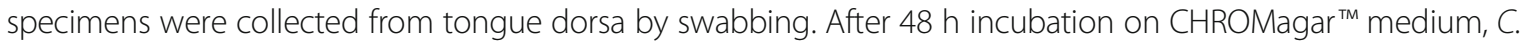
albicans and non-albicans were identified by the morphology and pigmentation of the colonies. Oral and systemic health check-ups were performed to assess the following: number of remaining teeth and periodontal status, oral hygiene, use of dentures, obesity, hypertension, hyperlipidemia, and hyperglycemia. A questionnaire addressed lifestyle, medications, and living conditions. Using the variables above, the relative factors involved in the colonization and the amounts of each type of Candida were determined.

Results: C. albicans and non-albicans were detected in 142 (53.4\%) and 63 (23.7\%) participants, respectively. Multinomial logistic regression analyses revealed that the significant factors of colonization by $C$. albicans were "having decayed teeth" and "relocation from home". Factors related to non-albicans colonization were "age over 80 years", "number of remaining teeth", "use of dentures", and "obesity". On the contrary, none of the parameters were related to the amount of non-albicans in the carrier, and the amount of C. albicans was significantly associated with "number of teeth" and "hypertension".

Conclusions: Prevalence-related factors differed between C. albicans and non-albicans colonization. In addition, other than oral status, systemic and living conditions affected the prevalence of both C. albicans and non-albicans in elderly people living in a post-disaster area.
\end{abstract}

Keywords: Candida albicans, Non-albicans, Elderly, Post-disaster area

\footnotetext{
* Correspondence: mkishi@iwate-med.ac.jp

${ }^{1}$ Division of Preventive Dentistry, Department of Oral Medicine, School of

Dentistry Iwate Medical University, 1-3-27 Chuo-dori, Morioka, Iwate

020-8505, Japan

Full list of author information is available at the end of the article
}

\section{$\int$ Biomed Central}

(c) The Author(s). 2017 Open Access This article is distributed under the terms of the Creative Commons Attribution 4.0 International License (http://creativecommons.org/licenses/by/4.0/), which permits unrestricted use, distribution, and reproduction in any medium, provided you give appropriate credit to the original author(s) and the source, provide a link to the Creative Commons license, and indicate if changes were made. The Creative Commons Public Domain Dedication waiver (http://creativecommons.org/publicdomain/zero/1.0/) applies to the data made available in this article, unless otherwise stated. 


\section{Background}

Over 40 species of Candida yeasts, the most common of which is Candida albicans, can cause infections in humans. In addition, C. tropicalis, C. parapsilosis, and $C$. glabrata are major pathogenic Candida species collectively referred to as non-albicans [1-4]. Several Candida species colonize mucosal surfaces in the oral cavity, digestive tract, and vagina. Candida are normal commensal organisms of the mouth and generally cause no problems in healthy people. In the general population, carriage rates are reported to be in the range 3 to $75 \%$ without any symptoms [5]. However, the overgrowth of Candida in oropharyngeal or esophageal mucosa causes a burning sensation, taste disorders, severe mucositis, or dysphagia and results in poor nutrition. From these symptoms, oral candidiasis is the most common human fungal infection, especially in the elderly or hospitalized patients [5-7]. Once the oral cavity is colonized, it becomes easier for the yeasts to reach the respiratory system, and since it is a commensal species of the gut lumen and the cutaneous surfaces, the colonization index is increasing $[7,8]$. Thus, oral Candida poses a risk of systemic disorders as well as local mucosal infections in the elderly. Previous studies indicate that the risk factors involved in oral Candida colonization in the elderly include wearing dentures, poor oral/denture hygiene, and low local saliva flow [9-15]. Furthermore, elderly individuals are at risk of overgrowth of Candida due to several predisposing factors, such as systemic disease, decreased immune function, and the use of various medicines [7, 10,16-19]. Therefore, the risks of Candida colonization should be clarified in terms of both systemic and oral conditions, especially in elderly people who are likely to harbor Candida and are more likely to receive hospital care.

In 2011, Japan experienced the Great East Japan Earthquake, a catastrophic disaster. Most of the severely damaged areas were rural and consisted of already aging communities compared with other areas of Japan, which has one of the largest aging populations in the world [20]. Moreover, the rate of growth of the elderly population has rapidly increased due to the outflow of the younger generation in post-disaster areas, and a considerable number of elderly people are still displaced from their homes 5 years after the disaster [21]. The extraordinary lifestyle after the disaster has had negative effects on systemic, mental, and oral health [22-24], and could affect the prevalence of oral Candida colonization in elderly community dwellers in the disaster areas.

The primary aim of this study was to assess the prevalence of oral Candida colonization and distinguish nonalbicans from $C$. albicans in elderly community dwellers of a post-disaster area because non-albicans has recently been detected more frequently [25].
A second aim was to identify the factors associated with oral Candida colonization among demographic, oral, and systemic conditions, lifestyle, medication status, and living conditions.

\section{Methods}

\section{Study design and population}

This was a cross-sectional study involving clinical surveys, a questionnaire, and microbiological examinations using culture methods. The participants were community dwellers aged 60 years or over in Otsuchi, located on the Pacific Coast of Iwate Prefecture, which experienced some of the most severe damage from the Great East Japan Earthquake and Tsunami. However, the area suffered no effects of radiation from the Fukushima Daiichi Nuclear Disaster because the distance from the power station was over $200 \mathrm{~km}$. In 2014, from individuals who attended an annual oral health checkup that we had carried out since 2011, 266 candidates aged 60 years or older who lived in the tsunami inundated area at the disaster site, were randomly recruited to an additional examination to detect Candida species (Fig. 1). Detailed methods of initial subject recruitment were reported in our previous paper [24]. All the candidates gave informed consent to participate in this study after receiving sufficient information. The mean age and standard deviation of our participants were 72.3 and 7.0. None of the participants used antibiotics, or antifungal medications during the survey.

The study protocol was approved by the Medical Ethics Committee of Iwate Medical University School of Medicine (H23-69) and School of Dentistry (01214).

\section{Sample collection and identification of Candida species}

Oral samples were collected by swabbing 10 times vertically from the circumvallate papillae to the tip of the tongue with a sterile cotton swab. Each swab was immediately immersed into $2 \mathrm{~mL}$ of sterile phosphate buffered

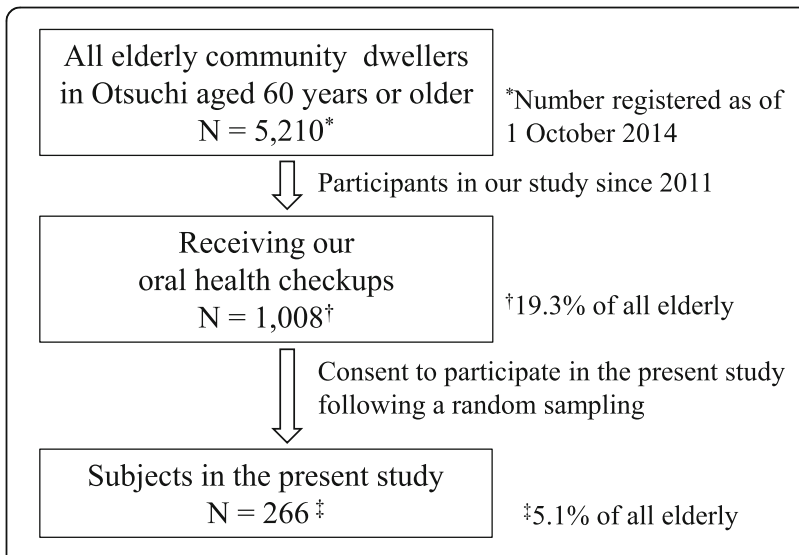

Fig. 1 Sample selection and description of the study population 
saline (PBS, pH 7.4) in a stock tube and stored on ice until inoculation. After stirring with a Vortex Mixer, the cotton swab was removed from the PBS and the suspension was inoculated onto a CHROMagar Candida ${ }^{\mathrm{TM}}$ plate (CHROMagar Microbiology, Paris, France) within $8 \mathrm{~h}$ of collection, because we confirmed in our preliminary study that the time elapsed would not affect the culture results. After $48 \mathrm{~h}$ of incubation, all colonies observed on CHROMagar ${ }^{\text {Tw }}$ were identified by their morphology and pigmentation according to the manufacturer's instructions and a previous study [26]. In addition, the colony forming unit/mL (CFU) was calculated for both $C$. albicans and non-albicans.

\section{Oral examinations}

Oral examinations were performed by a skilled dentist. Dental caries status was assessed according to the World Health Organization (WHO) method [27] with some modifications as follows: (1) a tooth with treated or untreated root caries was recorded as a filled or decayed, and (2) a remaining root without a crown was counted as a present tooth. Periodontal conditions were assessed using the Community Periodontal Index (CPI) procedures and diagnostic criteria, which are also recommended by the WHO. In addition, oral hygiene status was assessed as one of three grades: poor, fair, or good. Information on denture use was obtained from both oral examination and interview. In later analyses, participants with a CPI code of 3 or 4 (having periodontal pockets) were summarized, and oral hygiene status was categorized as poor or other status.

\section{Systemic data}

Body mass index (BMI, $\mathrm{kg} / \mathrm{m}^{2}$ ), blood pressure (BP, $\mathrm{mmHg}$ ) and biochemical data, including high-density lipoprotein cholesterol (HDLC; $\mathrm{mg} / \mathrm{dL}$ ), low-density lipoprotein cholesterol (LDLC; $\mathrm{mg} / \mathrm{dL}$ ), and glycated hemoglobin (HbA1c, \%) were obtained from the results of health check-ups that the municipality supplied as a public health service. The health check-up was performed according to a Japanese national guideline (the Specific Health Checkups) [28]. Participants were placed in one of two groups depending on the measurements, using the following cutoff values: BMI $\geq 25$ (obesity), systolic $\mathrm{BP} \geq 140$ or diastolic $\mathrm{BP} \geq 90$ (hypertension), HDLC $\leq 34$ or LDLC $\geq 140$ (dyslipidemia), and HbA1c $\geq 5.6 \%$ (hyperglycemia).

\section{Self-reported data}

Self-report questionnaires were administrated to assess lifestyle (smoking and drinking status), daily medications (antihypertension drugs, diabetes drugs, hypnotics), and whether the participants' current accommodation was the same as that before the disaster (relocation from home). The choices of answers for smoking status were current smoker, past smoker, and non-smoker. We categorized those choices into current smoker or other in our statistical analyses. Similarly, choices for drinking status (daily drinker, occasional drinker, and no drinker) were summarized into daily drinker or other.

\section{Statistical analyses}

The prevalence of Candida was examined in all 266 participants. In subsequent factor analyses, two participants were excluded because certain data was missing. To determine the factors related with colonization of $C$. albicans or non-albicans, we assessed risk measurements by multinomial logistic regression models followed by crude analyses using Chi-squared tests. To compare the amounts of Candida species between the parameters, the MannWhitney $U$ test or Kruskal-Wallis test was used, since the distribution of Candida amounts were non-normal after logarithmic transformation. Post hoc multiple comparisons after the Kruskal-Wallis test were performed using the Mann-Whitney $U$ test with Bonferroni correction. To reveal the relationship between number of remaining teeth and $\log$ CFUs of C. albicans and non-albicans, Spearman's rank correlation analysis and logistic regression analysis using a cutoff value $(\log \mathrm{CFU}=1.5)$ were performed. A two-sided $p$-value of less than 0.05 was considered statistically significant. All statistical analyses were conducted using the software program SPSS version 24.0 for Windows (IBM).

\section{Results}

Prevalence of $C$. albicans and non-albicans

Candida species in tongue dorsa were detected in 162 participants $(60.9 \%)$. C. albicans was the most frequently detected in $(n=142 ; 53.4 \%)$ participants, followed by $C$. glabrata $(n=60 ; 22.6 \%), C$. tropicalis $(n=5 ; 1.8 \%)$, and C. parapsilosis $(n=2 ; 0.7 \%)$. C. krusei were not detected in any of our participants. At least one non-albicans species were detected in $63(23.7 \%)$ of the participants (Table 1). In addition, $61.1 \%$ and $11.7 \%$ of Candida carriers exclusively harbored either C. albicans or C. glabrata, respectively. A combination of $C$. albicans and $C$. glabrata was found in $23.5 \%$ of Candida carriers, and other combinations were found in only $3.7 \%$.

\section{Factors associated with colonization by C. albicans and non-albicans}

Tables 2 and 3 show the significant factors related to colonization by $C$. albicans and non-albicans in the demographic data and the parameters measured in this study (see Additional file: 1 for complete data). A crude odds ratio (COR) with a $95 \%$ confidence interval (CI) was calculated from a bi-variable Chi-squared test, and the adjusted odds ratio (AOR) with 95\% CI was 
Table 1 Detection rates of Candida in elderly community dwellers $(n=266)$ in a post-disaster area

\begin{tabular}{llll}
\hline Candida spp. & $\begin{array}{l}\text { Number of } \\
\text { carriers }\end{array}$ & $\begin{array}{l}\text { \% of entire } \\
\text { subjects }\end{array}$ & $\begin{array}{l}\text { \% of carriers of } \\
\text { any Candida }\end{array}$ \\
\hline C. albicans & 142 & 53.4 & 87.7 \\
C. glabrata & 60 & 22.6 & 37.0 \\
C. tropicalis & 5 & 1.8 & 3.1 \\
C. parapsilosis & 2 & 0.7 & 1.2 \\
C. krusei & 0 & 0 & 0 \\
Any non-albicans & 63 & 23.7 & 38.9 \\
Any Candida spp. & 162 & 60.9 & - \\
\hline
\end{tabular}

calculated from multinomial logistic regression analysis in which the variables were all adjusted for each other. In the bi-variable models, participants "having one or more decayed teeth", "poor oral hygiene" and "relocation from home" harbored C. albicans at a significantly higher frequency $(\mathrm{COR}=3.35 ; 95 \% \mathrm{CI}=1.70-6.63$, $\mathrm{COR}$ $=2.42 ; 95 \% \mathrm{CI}=1.18-4.99$ and $\mathrm{COR}=1.45 ; 95 \% \mathrm{CI}=$ 1.06-1.99, respectively). For colonization of non-albicans, "over 80 years old", "less than 20 remaining teeth/ edentulous", "use of dentures" and "obesity" were significant factors associated with colonization $(\mathrm{COR}=2.21$; $95 \% \mathrm{CI}=1.36-5.34, \quad \mathrm{COR}=1.84 / 2.39 ; 95 \% \mathrm{CI}=1.51-$ $2.42 / 1.83-3.12, \quad \mathrm{COR}=1.56 ; 95 \% \mathrm{CI}=1.37-1.77$ and $\mathrm{COR}=1.45 ; 95 \% \mathrm{CI}=1.06-2.00$, respectively). In the multivariable models, "having one or more decayed teeth" and "relocation from home" were significant factors for colonization by $C$. albicans similarly to bivariable analyses, although "poor oral hygiene" was not significant $(\mathrm{AOR}=3.51 ; 95 \% \mathrm{CI}=1.60-7.67, \mathrm{AOR}=2.17$; $95 \% \mathrm{CI}=1.25-3.78$ and $\mathrm{AOR}=2.01 ; 95 \% \mathrm{CI}=0.77-5.24$, respectively). The same factors were significant to colonization by non-albicans in bi-variable analyses, excluding "less than 20 teeth" (AOR $=3.37$; 95\% CI $=0.88$ 12.9). In addition, the odds ratios of "over 80 years old", "edentulous", "use of dentures" and "obesity" were raised after adjustment for each other $(\mathrm{AOR}=2.58 ; 95 \% \mathrm{CI}=$ $1.12-5.71, \mathrm{AOR}=5.99 ; 95 \% \mathrm{CI}=1.37-26.3, \mathrm{AOR}=4.02$; $95 \% \mathrm{CI}=1.05-15.4$ and $\mathrm{AOR}=2.25 ; 95 \% \mathrm{CI}=1.15-4.40$, respectively).

\section{Factors related to the amounts of $C$. albicans and non-albicans among the carriers}

We compared the amounts (log CFU) of Candida between same parameters as the examination for colonizationrelated factors of each Candida carrier. Larger amounts of C. albicans were detected in edentulous subjects than in dentate subjects among C. albicans carriers. In addition, participants with hypertension and a daily drinking habit harbored greater amounts of $C$. albicans. However, the amounts of non-albicans among the carriers were no different between any of the categories (Table 4).

\section{Correlation between amounts of $C$. albicans and non-albicans with number of remaining teeth in denture wearers}

Spearman's correlation coefficient between amounts ( $\log$ CFU) of Candida, number of remaining teeth and age were examined in each Candida carrier wearing a denture (Table 5). There were significant correlations between $\log$ CFU and number of remaining teeth in both groups of carriers $(\rho=-0.40, p$-value $<0.01$ for $C$. albicans; $\rho=-0.38, p$-value $<0.01$ for non-albicans), although we also found significant relationships between age and number of remaining teeth $(\rho=-0.34, p$-value $<0.01$ for C. albicans; $\rho=-0.40, p$-value $<0.01$ for non-albicans). To adjust for possible confounders, we applied logistic regression models using a cutoff value. In these models, the dependent variable was a high amount of each type of Candida $(\mathrm{CFU} \geq 1.5)$ and the independent variables were number of remaining teeth and age in years. As Table 6 shows, after a stepwise procedure, the number of remaining teeth was selected as a significant factor of high amounts of both C. albicans and non-albicans (adjusted

Table 2 Significant factors related to colonization of C. albicans in surveyed parameters in this study $(N=264)$

\begin{tabular}{|c|c|c|c|c|}
\hline Parameter & $n$ & COR (95\% Cl) $p$-value & AOR $(95 \% \mathrm{Cl}) p$-value & Number of positive (\%) \\
\hline \multicolumn{5}{|c|}{ Having one or more decayed teeth } \\
\hline Yes & 53 & $3.35(1.70-6.63)<0.01$ & $3.51(1.60-7.67)<0.01$ & $40(75.5)$ \\
\hline $\mathrm{No}^{\mathrm{a}}$ & 211 & 1.00 & 1.00 & $101(47.9)$ \\
\hline \multicolumn{5}{|l|}{ Oral hygiene } \\
\hline Poor & 34 & $2.42(1.18-4.99)<0.01$ & $2.01(0.77-5.24) 0.15$ & $25(73.5)$ \\
\hline Fair/Good & 230 & & 1.00 & $116(50.4)$ \\
\hline \multicolumn{5}{|c|}{ Relocation from home } \\
\hline Yes & 104 & $1.45(1.06-1.99) 0.02$ & $2.17(1.25-3.78)<0.01$ & $65(62.5)$ \\
\hline No & 160 & 1.00 & 1.00 & $76(47.5)$ \\
\hline
\end{tabular}

Parameters include significant factors in either bivariate or multivariate analysis, COR crude odds ratio from bivariate analysis, $A O R$ adjusted odds ratio from multinomial logistic regression analysis; $\mathrm{Cl}$ confidence interval

${ }^{a}$ The reference categories are in the last row for all explanatory variables 
Table 3 Significant factors related to colonization of Non-albicans in surveyed parameters in this study $(N=264)$

\begin{tabular}{|c|c|c|c|c|}
\hline & $n$ & COR (95\% Cl) $p$-value & AOR $(95 \% \mathrm{Cl}) p$-value & Number of positive (\%) \\
\hline \multicolumn{5}{|l|}{ Age (in years) } \\
\hline$\geq 80$ & 44 & $2.21(1.36-5.34)<0.01$ & $2.58(1.12-5.71) 0.02$ & $18(40.9)$ \\
\hline$<80^{\mathrm{a}}$ & 220 & 1.00 & 1.00 & $45(20.5)$ \\
\hline \multicolumn{5}{|c|}{ Number of remaining teeth } \\
\hline Edentulous & 73 & $2.39(1.83-3.12)<0.01$ & $5.99(1.37-26.3) 0.02$ & $27(37.0)$ \\
\hline $1-19$ & 107 & $1.84(1.51-2.42)<0.01$ & $3.37(0.88-12.9) 0.08$ & $32(29.9)$ \\
\hline 20 or more & 84 & 1.00 & 1.00 & $4(4.8)$ \\
\hline \multicolumn{5}{|c|}{ Use of dentures } \\
\hline Yes & 180 & $1.56(1.37-1.77)<0.01$ & $4.02(1.05-15.4) 0.04$ & $59(32.8)$ \\
\hline No & 84 & 1.00 & 1.00 & $4(4.8)$ \\
\hline \multicolumn{5}{|c|}{ Obesity (BMI $\geq 25$ ) } \\
\hline Yes & 99 & $1.45(1.06-2.00) 0.04$ & $2.25(1.15-4.40) 0.02$ & $31(31.3)$ \\
\hline No & 165 & 1.00 & 1.00 & $32(19.4)$ \\
\hline
\end{tabular}

Parameters include significant factors in either bivariate or multivariate analysis, COR crude odds ratio from bivariate analysis, $A O R$ adjusted odds ratio from multinomial logistic regression analysis; $\mathrm{Cl}$ confidence interval

${ }^{a}$ The reference categories are in the last row for all explanatory variables

partial regression coefficient $=0.94 ; 95 \% \mathrm{CI}=0.88-0.99$ and adjusted partial regression coefficient $=0.91 ; 95 \%$ $\mathrm{CI}=0.83-0.98)$.

\section{Discussion}

In this study, the subjects were community dwellers in a post-disaster area. There have been several reports on prevalence of Candida species, mainly in institutionalized elderly, or in patients with HIV, carcinoma, or diabetes $[9,29-34]$. However, information on the carriage rates of oral Candida in community dwellers is limited. Wang et al. [18] reported that $68.6 \%$ of communitydwelling elderly Japanese people aged 75 years were positive for total Candida. In addition, Goto et al. [35] reported prevalence rates of $63.6 \%$ for total Candida, and $53.4 \%$ for C. albicans in an elderly population aged 65-74. These rates are very close to those of our subjects $(60.9 \%$ for total Candida and $53.4 \%$ for C. albicans); however, Candida prevalence varies widely in the oral cavities of healthy individuals according to several previous studies $[5,25,36]$. The factors associated with Candida colonization in the oral cavities of elderly people include age, use of dentures, poor oral/denture hygiene, and low saliva flow, in addition to systemic diseases [9-15]. The similar overall carriage rates of Candida found in these studies and ours in similarly aged Japanese elderly people may indicate that differences among oral or systemic conditions do not affect the colonization status of Candida even in post-disaster areas.

We found differences among the factors associated with oral colonization between C. albicans and non-albicans (Tables 2 and 3). The oral factors affecting colonization presented in previous studies, such as use of dentures or number of remaining teeth, were associated exclusively with non-albicans in our subjects. On the contrary, quantitative analysis revealed that none of the parameters were associated with the colony counts of non-albicans, although edentulous subjects harbored significantly greater amounts of C. albicans. In addition, denture users tended to have a greater amount of $C$. albicans although this was not statistically significant (Table 4). The dissimilarities between Candida species both in our qualitative and quantitative analyses suggested differences in the time of colonization by $C$. albicans and non-albicans. Elderly subjects might harbor stable non-progressive colonization by $C$ albicans, while non-albicans colonization could be progressive. Therefore, despite the number of remaining teeth, or use of dentures being common factors in the prevalence of both Candida species, they might have largely affected colonization by non-albicans and growth of C. albicans, at least in our subjects. On the other hand, colonization by $C$. albicans related significantly to "having one or more decayed teeth". The relationship between Candida carriage and dental caries status has mainly been reported in children [37-39]. In these studies, C. albicans or total Candida carriage was generally associated with the prevalence of dental caries. However, race, age, country, and the oral status of the subjects in these previous studies were very different from our study, which makes it difficult for us to compare our results. In addition, the previous studies aimed to clarify the etiology of Candida in dental caries. Rather, we presumed that "having decayed teeth" was a consequence of poor living conditions after the disaster. Affected individuals were exempt from medical insurance premiums and 
Table 4 Comparisons of colony amounts of Candida species by demographic characteristics, oral conditions, systemic conditions, lifestyle, medications, and relocation from home in carriers

\begin{tabular}{|c|c|c|c|c|c|c|}
\hline & \multicolumn{3}{|l|}{ C. albicans } & \multicolumn{3}{|l|}{ Non-albicans } \\
\hline & No. of carriers & Mean log CFU (SD) & $p$-value ${ }^{a}$ & No. of carriers & Mean log CFU (SD) & $p$-value \\
\hline \multicolumn{7}{|l|}{ Sex } \\
\hline Female & 78 & $1.44(0.65)$ & 0.72 & 42 & $1.91(0.92)$ & 0.63 \\
\hline Male $^{a}$ & 63 & $1.44(0.66)$ & & 21 & $1.73(0.74)$ & \\
\hline \multicolumn{7}{|c|}{ Age (in years) } \\
\hline$\geq 80$ & 29 & $1.59(0.74)$ & 0.24 & 18 & $1.87(0.88)$ & 0.84 \\
\hline$<80$ & 112 & $1.40(0.63)$ & & 45 & $1.85(0.86)$ & \\
\hline \multicolumn{7}{|c|}{ Having one or more decayed teeth } \\
\hline Yes & 40 & $1.45(0.60)$ & 0.77 & 13 & $1.56(0.62)$ & 0.31 \\
\hline No & 101 & $1.43(0.68)$ & & 50 & $1.93(0.90)$ & \\
\hline \multicolumn{7}{|c|}{ Number of remaining teeth } \\
\hline Edentulous & 33 & $1.88(0.67)$ & $<0.01^{\mathrm{b}}$ & 27 & $2.17(0.93)$ & 0.09 \\
\hline $1-19$ & 60 & $1.35(0.61)$ & & 32 & $1.62(0.78)$ & \\
\hline 20 or more & 48 & $1.25(0.56)$ & & 4 & $1.55(0.24)$ & \\
\hline \multicolumn{7}{|c|}{ Having periodontal pockets } \\
\hline Yes & 41 & $1.32(0.66)$ & 0.16 & 14 & $1.69(0.71)$ & 0.55 \\
\hline No & 100 & $1.49(0.65)$ & & 49 & $1.90(0.60)$ & \\
\hline \multicolumn{7}{|l|}{ Oral hygiene } \\
\hline Poor & 25 & $1.39(0.65)$ & 0.70 & 8 & $1.69(0.73)$ & 0.75 \\
\hline Fair/Good & 116 & $1.45(0.66)$ & & 55 & $1.88(0.88)$ & \\
\hline \multicolumn{7}{|l|}{ Use of denture } \\
\hline Yes & 93 & $1.52(0.68)$ & 0.07 & 59 & $1.88(0.88)$ & 0.49 \\
\hline No & 48 & $1.29(0.58)$ & & 4 & $1.45(0.40)$ & \\
\hline \multicolumn{7}{|c|}{ Obesity (BMI $\geq 25$ ) } \\
\hline Yes & 11 & $1.42(0.72)$ & 0.86 & 31 & $1.93(0.89)$ & 0.50 \\
\hline No & 130 & $1.44(0.65)$ & & 32 & $1.78(0.84)$ & \\
\hline \multicolumn{7}{|c|}{ Hypertension (systolic BP $\geq 140$ or diastolic BP $\geq 90$ ) } \\
\hline Yes & 38 & $1.61(0.63)$ & 0.04 & 24 & $1.84(0.77)$ & 0.96 \\
\hline No & 103 & $1.37(0.65)$ & & 39 & $1.86(0.92)$ & \\
\hline \multicolumn{7}{|c|}{ Dyslipidemia (HDLC $\leq 34$ or LDLC $\geq 140$ ) } \\
\hline Yes & 37 & $1.31(0.57)$ & 0.20 & 14 & $1.90(0.92)$ & 0.99 \\
\hline No & 104 & $1.48(0.68)$ & & 49 & $1.84(0.85)$ & \\
\hline \multicolumn{7}{|c|}{ Hyperglycemia (HbA1c $\geq 5.6$ ) } \\
\hline Yes & 31 & $1.58(0.67)$ & 0.14 & 17 & $2.07(0.71)$ & 0.50 \\
\hline No & 110 & $1.40(0.65)$ & & 46 & $1.77(0.90)$ & \\
\hline \multicolumn{7}{|c|}{ Current smoker } \\
\hline Yes & 11 & $1.42(0.72)$ & 0.86 & 5 & $1.63(0.88)$ & 0.58 \\
\hline No & 130 & $1.44(0.65)$ & & 58 & $1.87(0.86)$ & \\
\hline \multicolumn{7}{|l|}{ Daily drinker } \\
\hline Yes & 19 & $1.72(0.67)$ & 0.40 & 7 & $1.81(1.26)$ & 0.54 \\
\hline No & 122 & 1.39 (0.64) & & 56 & $1.86(0.81)$ & \\
\hline
\end{tabular}


Table 4 Comparisons of colony amounts of Candida species by demographic characteristics, oral conditions, systemic conditions, lifestyle, medications, and relocation from home in carriers (Continued)

\begin{tabular}{|c|c|c|c|c|c|c|}
\hline \multicolumn{7}{|c|}{ Taking antihypertensive drugs } \\
\hline Yes & 83 & $1.48(0.65)$ & \multirow[t]{2}{*}{0.30} & 38 & $1.98(0.83)$ & \multirow[t]{2}{*}{0.09} \\
\hline No & 58 & $1.37(0.66)$ & & 25 & $1.65(0.88)$ & \\
\hline \multicolumn{7}{|c|}{ Taking diabetes drugs } \\
\hline Yes & 8 & $1.45(0.60)$ & \multirow[t]{2}{*}{0.80} & 5 & $2.11(0.82)$ & \multirow[t]{2}{*}{0.41} \\
\hline No & 133 & $1.43(0.66)$ & & 58 & $1.83(0.87)$ & \\
\hline \multicolumn{7}{|c|}{ Taking hypnotics } \\
\hline Yes & 25 & $1.49(0.76)$ & \multirow[t]{2}{*}{0.81} & 12 & $2.15(0.79)$ & \multirow[t]{2}{*}{0.14} \\
\hline No & 116 & $1.42(0.63)$ & & 51 & $1.78(0.87)$ & \\
\hline \multicolumn{7}{|c|}{ Relocation from home } \\
\hline Yes & 65 & $1.40(0.65)$ & \multirow[t]{2}{*}{0.50} & 25 & $1.82(0.84)$ & \multirow[t]{2}{*}{0.72} \\
\hline No & 76 & $1.47(0.66)$ & & 38 & $1.88(0.89)$ & \\
\hline
\end{tabular}

${ }^{a}$ Statistical differences were examined by Mann-Whitney $U$ test for bivariate combinations, and Kruskal-Wallis test for comparison of 3 groups

${ }^{b}$ Post-hoc multiple comparisons using Bonferroni adjustment indicated that there was statistical significance between edentulous and others

payments at medical institution counters, including dental facilities, by the Japanese government and the local municipalities [40]. However, despite the existence of these services, subjects with decayed teeth did not appear to have received dental care for some reason, perhaps due to lack of transportation, social networks, and so on. C. albicans was also found more frequently in subjects living in a different house from their own home (many of them were in temporary housing). Moreover, significant trends in the prevalence of Candida species were also found in general health conditions such as obesity and colonization by non-albicans, and hypertension and colony counts of C. albicans in C. albicans carriers. The reasons are unclear, however, such symptoms tend to originate from an unhealthy lifestyle. Therefore, our findings suggested that living conditions and systemic disorders derived from lifestyle other than oral factors could affect the prevalence of Candida elderly people living in post-disaster areas.

Analyses of denture wearers harboring C. albicans or non-albicans revealed negative correlations between number of remaining teeth and the colony counts of both $C$. albicans and non-albicans after adjusting for the confounders number of remaining teeth and age (Table 5 and 6). Thus, positive correlations were found between the number of missing teeth and the quantities of Candida. Accordingly, dentures covering large areas of oral mucosa could be a risk factor for high amounts of both $C$. albicans and non-albicans. Although studies on the relationship between dentures and the amount of Candida, or denture size and frequency of Candida colonization have occasionally been reported [41, 42], our research might be the first study on denture size and the amount of Candida, especially in subjects without symptoms. However, it is unclear whether the colony counts of Candida is a risk factor for the incidence of candidiasis. Further investigation into the significance of amounts of the colonization of Candida is needed.

This study had several limitations. First, the number of subjects represented a small proportion of the entire community (population of 60 years or older was 5,210 on 1 October 2014, according to a government report). However, our subjects were probability samples recruited from all participants who took part in our dental health check-ups. We believe they represented all residents to a reliable degree because the total number of participants $(n=1,008$, Fig. 1$)$ were sufficient to represent the entire elderly population. Second, we did

Table 5 Relationships between colony amounts of Candida species, age, and number of remaining teeth in carriers wearing dentures

\begin{tabular}{|c|c|c|c|c|c|c|}
\hline & \multicolumn{3}{|c|}{ C. albicans ( $n=93$ ) } & \multicolumn{3}{|c|}{ Non-albicans ( $n=59)$} \\
\hline & Log CFU & Number of remaining teeth & Age & Log CFU & Number of remaining teeth & Age \\
\hline Log CFU & & $-0.40(<0.01)$ & $-0.18(0.09)$ & & $-0.38(<0.01)$ & $0.10(0.45)$ \\
\hline Number of remaining teeth & $-0.40(<0.01)$ & & $-0.34(<0.01)$ & $-0.38(<0.01)$ & & $-0.40(<0.01)$ \\
\hline Age & $-0.18(0.09)$ & $-0.34(<0.01)$ & & $0.10(0.45)$ & $-0.40(<0.01)$ & \\
\hline
\end{tabular}

Value shows Spearman's correlation coefficient ( $p$-value) 
Table 6 Relationship between age, number of remaining teeth, and colony amounts of Candida by logistic regression models ${ }^{\mathrm{a}}$

\begin{tabular}{|c|c|c|c|c|c|}
\hline \multicolumn{6}{|l|}{ C. albicans $(n=93)$} \\
\hline & Variable & Crude partial regression coefficient & Adjusted partial regression coefficient & $95 \% \mathrm{Cl}$ & $p$-value \\
\hline \multirow[t]{2}{*}{ First step } & Number of remaining teeth & -0.06 & 0.94 & $0.53-0.94$ & 0.053 \\
\hline & Age & 0.01 & 1.02 & $0.95-1.08$ & 0.65 \\
\hline Second (final) step & Number of remaining teeth & -0.07 & 0.94 & $0.88-0.99$ & 0.03 \\
\hline \multicolumn{6}{|l|}{ Non- albicans $(n=59)$} \\
\hline & Variable & Partial regression coefficient & Adjusted partial regression coefficient & $95 \% \mathrm{Cl}$ & $p$-value \\
\hline \multirow[t]{2}{*}{ First step } & Number of remaining teeth & -0.11 & 0.90 & $0.82-0.98$ & 0.02 \\
\hline & Age & -0.02 & 0.62 & $0.90-1.06$ & 0.62 \\
\hline Second (final) step & Number of remaining teeth & -0.10 & 0.91 & $0.83-0.98$ & 0.02 \\
\hline
\end{tabular}

${ }^{a}$ Dependent variable: $\log$ CFU $\geq 1.5 ; 1, \log$ CFU $<1.5 ; 0$

not assess dryness of the mouth since this could not be conducted in our field survey. Based on the previous studies, this factor is frequently associated with Candida prevalence. Our models accounting for the prevalence of Candida would have been more accurate if this factor had been included. Third, being a cross-sectional study, it essentially lacked data from the affected region before the disaster, although we expect to provide supplemental data in a follow-up study. Finally, whether our findings are specific to post-disaster areas remains unclear given the lack of comparative data from non-disaster situation. This study was conducted as part of a large-scale cohort study aimed at supporting victims of the Great East Japan Earthquake. Since the research design did not include control areas at the beginning of the study in 2011 [22-24], it was not possible to establish control areas during the present survey because of time and budget constraints. However, compared to some previous studies for similarly aged elderly people living in ordinary conditions, our results partially identified relative post-disaster risks for the prevalence of Candida and can be generalized to some degree.

\section{Conclusions}

In our participants, the factors related to colonization or amounts differed between $C$. albicans and nonalbicans. It is suggested that there is a difference in the time of colonization between Candida species. In addition, the amounts of both Candida types were related to greater numbers of missing teeth. Perhaps the amount of Candida on the tongue dorsa increased with increases in size of the area covered by the denture. Further, systemic and living conditions, other than oral status, could affect the prevalence of both $C$. albicans and non-albicans among elderly people living in a post-disaster area.

\section{Additional file}

Additional file 1: Relationships of demographic character, oral conditions, systemic conditions, lifestyle, medications and relocation from home with colonization of $C$. albicans and non-albicans ( $N=$ 264). Results of multinomial logistic regression analysis for colonization of C. albicans and non-albicans. Relationships of C. albicans and non-albicans colonization with all independent variables examined in this study are presented in this table. (DOCX $22 \mathrm{~kb}$ )

\section{Abbreviations}

AOR: Adjusted odds ratio; BMI: Body mass index; BP: Blood pressure; CFU: Colony forming unit/mL; Cl: Confidential interval; COR: Crude odds ratio; CPI: Community Periodontal Index; HbA1c: Glycosylated hemoglobin; HDLC: High-density lipoprotein cholesterol; LDLC: Low-density lipoprotein cholesterol; SD: Standard deviation; WHO: World Health Organization

\section{Acknowledgements}

We sincerely thank all of the staff in the town of Otsuchi for their support. We also thank Dr. Yuki Yonekura for useful advice while writing the manuscript.

\section{Funding}

The current study was supported by Grant-in-Aid from the Ministry of Health, Labor and Welfare, Health and Labor Sciences Research Grants, Japan [H23-Tokubetsu-Shitei-002; H24-Kenki-Shitei-001, H25-Kenki-Shitei-001(Fukkou)], and JSPS KAKENHI Grant number 15 K11425. The funding body had no role in the study design, the collection, analysis, and interpretation of data, writing the manuscript, or in the decision to submit the manuscript for publication.

\section{Availability of data and materials}

The datasets obtained and/or analyzed during the current study are available from the corresponding author on reasonable request.

\section{Authors' contributions}

TS conceived this study, executed almost all parts of the survey, and drafted the manuscript. MK administered the study, and contributed to data analysis. MS contributed to data acquisition. HS administered data other than oral data. HM organized dental health survey team. KS, SK and AO planned and supervised this study. All authors read and approved the final manuscript.

\section{Competing interests}

The authors declare that they have no coompeting interest.

Consent for publication

Not applicable. 


\section{Ethics approval and consent to participate}

We obtained informed consent from all participants who took part in our study. This study protocol was approved by the Medical Ethics Committee of Iwate Medical University School of Medicine (H23-69) and School of Dentistry (01214).

\section{Author details}

'Division of Preventive Dentistry, Department of Oral Medicine, School of Dentistry Iwate Medical University, 1-3-27 Chuo-dori, Morioka, Iwate 020-8505, Japan. ${ }^{2}$ Department of Hygiene and Preventive Medicine, Iwate Medical University, School of Medicine, 2-1-1 Nishitokuta, Yahaba, Iwate 028-3694, Japan. ${ }^{3}$ Division of Dental Education, Department of Oral Medicine, School of Dentistry Iwate Medical University, 1-3-27 Chuo-dori, Morioka, Iwate 020-8505, Japan. ${ }^{4}$ Iwate Medical University, 19-1 Uchimaru, Morioka, Iwate 020-8505, Japan.

\section{Received: 24 September 2016 Accepted: 26 January 2017} Published online: 01 February 2017

\section{References}

1. Johnson EM. Rare and emerging Candida species. Curr Fungal Infect Rep. 2009:3:152-9.

2. Lucas VS. Association of psychotropic drugs, prevalence of denture-related stomatitis and oral conditions. Community Dent Oral Epidemiol. 1993;21: 313-6.

3. Arendorg TM, Walker DM. The prevalence and intra-oral distribution of Candida albicans in man. Arch Oral Biol. 1980;254:1-10.

4. Pfaller MA, Diekema DJ. Epidemiology of invasive candidiesis : a persistent public health problem. Clin Microbiol Rev. 2007;20:133-63.

5. Scully C, El-Kabir M, Samaranayake LP. Candida and oral candidosis : A review. Crit Rev Oral Biol Med. 1994;5:125-57.

6. Abu-Elteen $\mathrm{KH}, \mathrm{Rm}$ A-A. The prevalence of candida albicans populations in the mouths of complete denture wearers. New Microbiol. 1998;21:41-8.

7. Fanello S, Bouchara JP, Sauteron M, et al. Predictive value of oral colonization by Candida yeasts for the onset of a nosocomial infection in elderly hospitalized patients. J Med Microbiol. 2006;55:223-8.

8. Wisplinghoff H, Bischoff T, Tallent SM, Seifert $H$, Wenzel RP, Edmond MB. Nosocomial bloodstream infections in US hospitals: analysis of 24,179 cases from a prospective nationwide surveillance study. Clin Infect Dis. 2004;39: 309-17.

9. Grimoud AM, Marty N, Bocquet H, Andrieu S, Lodter JP, Chabanon G. Colonization of the oral cavity by candida species: risk factors in long-term geriatric care. J Oral Sci. 2003;45:51-5.

10. Dongari-Bagtzoglou A, Dwivedi P, loannidou E, Shaqman M, Hull D. Burleson J Oral Candida infection and colonization in solid organ transplant recipients. Oral Microbiol Immunol. 2009;24:249-54.

11. Budtz-Jörgensen E, Stenderup A, Grabowski M. An epidemiologic study of yeasts in elderly denture wearers. Community Dent Oral Epidemiol. 1975;3: 115-9.

12. Epstein JB, et al. Quantitative relationships between Candida albicans in saliva and the clinical status of human subjects. J Clin Microbiol. 1980;12: 475-6.

13. Navazesh $M$, et al. Relationship between salivary flow rates and Candida albicans counts. Oral Surg Oral Med Oral Pathol Oral Radiol Endod. 1995;80: 284-8.

14. Torres SR, et al. Relationship between salivary flow rates and Candida counts in subjects with xerostomia. Oral Surg Oral Med Oral Pathol Oral Radiol Endod. 2002;93:149-54.

15. Shimizu C, Kuriyama T, Williams DW, Karasawa T, Inoue K, Nakagawa K, Yamamoto E. Association of oral yeast carriage with specific host factors and altered mouth sensation. Oral Surg Oral Med Oral Pathol Oral Radiol Endod. 2008;105:445-51.

16. Aizen E, Feldman PA, Madeb R, Steinberg J, Merlin S, Sabo E, Perlov V, Srugo I. Candida albicans colonization of dental plaque in elderly dysphagic patients. Isr Med Assoc J. 2004;6:342-5.

17. Vargas $K G$, Joly S. Carriage frequency, intensity of carriage, and strains of oral yeast species vary in the progression to oral candidiasis in human immunodeficiency virus-positive individuals. J Clin Microbiol. 2002;40:341-50.

18. Wang J, Ohshima T, Yasunari U, Namikoshi S, Yoshihara A, Miyazaki H, Maeda N. The carriage of Candida species on the dorsal surface of the tongue: the correlation with the dental, periodontal and prosthetic status in elderly subjects. Gerodontology. 2006;23:157-63.

19. Yano A, Abe A, Aizawa F, Yamada H, Minami K, Matsui M, Kishi M. The effect of eating sea cucumber jelly on Candida load in the oral cavity of elderly individuals in a nursing home. Mar Drugs. 2013;11:4993-5007.

20. Annual Report on the Aging Society: 2016; Cabinet Office: Tokyo, Japan, 2016

21. Annual Report of Reconstruction Agency, Japanese government. 2016. http://www.reconstruction.go.jp/english/. Accessed 30 Jan 2017.

22. Takahashi S, Nakamura M, Yonekura Y, Tanno K, Sakata K, Ogawa A, Kobayashi S. Association between relocation and changes in cardiometabolic risk factors: a longitudinal study in tsunami survivors of the 2011 Great East Japan Earthquake. BMJ Open. 2016;12:6.

23. Yokoyama Y, Otsuka K, Kawakami N, Kobayashi S, Ogawa A, Tannno K, Onoda T, Yaegashi Y, Sakata K. Mental health and related factors after the Great East Japan earthquake and tsunami. PLOS ONE. 2014;24:9.

24. Kishi M, Aizawa F, Matsui M, Yokoyama Y, Abe A, Minami K, Suzuki R, Miura H, Sakata K, Ogawa A. Oral health-related quality of life and related factors among residents in a disaster area of the Great East Japan Earthquake and giant tsunami. Health Qual Life Outcomes. 2015;13:143.

25. Pfaller MA, Diekema DJ, Gibbs DL, et al. Results from the ARTEMIS DISK Global Antifungal Surveillance Study, 1997 to 2007; a 10.5 year analysis of susceptibilities of Candida species to fluconazole and voriconazole as determined by CLSI standardized disk diffusion. J Clin Microbiol. 2010;48: 1366-77.

26. Odds FC, Bernarets R. CHROMagar Candida, a new differential isolation medium for presumptive identification of clinically important Candida species. J Clin Microbiol. 1994;32:1923-9.

27. World Health Organization. Oral health surveys: basic methods. 4th ed. Geneva: World Health Organization; 1997.

28. Specific Health Checkups and Specific Health Guidance. http://www.mhlw. go.jp/english/wp/wp-hw3/dl/2-007.pdf. Accessed 30 Jan 2017.

29. Samaranayake LP, Wilkieson CA, Lamey PJ, MacFarlane TW. Oral disease in the elderly in long-term hospital care. Oral Dis. 1995;1:147-51.

30. Tortorano AM, Peman J, Bernhardt H, Klingspor L, Kibbler CC, Faure O, Biraghi E, Canton E, Zimmermann K, Seaton S, Grillot R. ECMM Working Group on Candidaemia. Epidemiology of candidaemia in Europe: results of 28-month European Confederation of Medical Mycology (ECMM) hospitalbased surveillance study. Eur J Clin Microbiol Infect Dis. 2004;23:317-22.

31. Sanitá PV, Pavarina AC, Giampaolo ET, Silva MM, Mima EG, Ribeiro DG, Vergani CE. Candida spp. prevalence in well controlled type 2 diabetic patients with denture stomatitis. Oral Surg Oral Med Oral Pathol Oral Radiol Endod. 2011;111:726-33.

32. Sangeorzan JA, Bradley SF, He X, Zarins LT, Ridenour GL, Tiballi RN, Kauffman CA. Epidemiology of oral candidiasis in HIV-infected patients: colonization, infection, treatment, and emergence of fluconazole resistance. Am J Med. 1994;97:339-46.

33. Wadhwa A, Kaur R, Agarwal SK, Jain S, Bhalla P. AIDS-related opportunistic mycoses seen in a tertiary care hospital in North India. J Med Microbiol. 2007:56:1101-6.

34. Belazi M, Velegraki A, Koussidou-Eremondi T, Andreadis D, Hini S, Arsenis G, Eliopoulou C, Destouni E, Antoniades D. Oral Candida isolates in patients undergoing radiotherapy for head and neck cancer: prevalence, azole susceptibility profiles and response to antifungal treatment. Oral Microbiol Immunol. 2004;19:347-51.

35. Goto J, Yamazaki Y, Sato J, Hata H, Oouchi M, Moriya S, Kitagawa Y. Research on carriage of Candida species in oral cavities of the homeindependent elderly. Hokkaido J Dent Sci. 2012;32:210-21 (in Japanese).

36. Oliver DE, Shillitoe EJ. Effects of smoking on the prevalence and intraoral distribution of Candida albicans. J Oral Pathol. 1984;13:265-70.

37. Srivastava B, Bhatia HP, Chaudhary V, Aggarwal A, Kumar Singh A, Gupta N. Comparative Evaluation of Oral Candida albicans Carriage in Children with and without Dental Caries: A Microbiological in vivo Study. Int J Clin Pediatr Dent. 2012;5:108-12

38. Al-Hebshi NN, Al-Maswary EA, Al-Hammadi ZO, Ghoname N. Salivary Candida species carriage patterns and their relation to caries experience among yemeni children. Oral Health Prev Dent. 2015;13:41-9.

39. De-la-Torre J, Marichalar-Mendia X, Varona-Barquin A, Marcos-Arias C, Eraso E, Aguirre-Urizar JM, Quindós G. Caries and Candida colonisation in adult patients in Basque Country (Spain). Mycoses. 2016. doi:10.1111/myc.12453 [Epub ahead of print]. 
40. Response to the Great East Japan Earthquake By the Ministry of Health, Labour and Welfare, the Government of Japan. 23 October 2012 Ministry of Health, Labour, and Welfare (MHLW) Government of Japan. http://www. mhlw.go.jp/bunya/kokusaigyomu/asean/2012/dl/Introduction_Dr.Yamauchi. pdf. Accessed 30 Jan 2017.

41. Kato T, Yamazaki Y, Sato J, Hata H, Oouchi M, Moriya S, Kitagawa Y. Reinvestigation of the carriage of Candida species in oral cavities of homeindependent elderly. Hokkaido J Dent Sci. 2013;33:121-39 (in Japanese).

42. Lockhart SR, Joly S, Vargas K, Swails-Wenger J, Enger L, Soll DR. Natural defenses against Candida colonization breakdown in the oral cavities of the elderly. J Dent Res. 1999;78:857-68.

Submit your next manuscript to BioMed Central and we will help you at every step:

- We accept pre-submission inquiries

- Our selector tool helps you to find the most relevant journal

- We provide round the clock customer support

- Convenient online submission

- Thorough peer review

- Inclusion in PubMed and all major indexing services

- Maximum visibility for your research

Submit your manuscript at www.biomedcentral.com/submit 